\title{
Systematic sampling during MRI-US fusion prostate biopsy can overcome errors of targeting-prospective single center experience after 300 cases in first biopsy setting
}

\author{
Emanuel Cata ${ }^{1,2 \#}$, Iulia Andras ${ }^{1,2}$, Matteo Ferro ${ }^{3}$, Pierre Kadula ${ }^{2 \#}$, Daniel Leucuta ${ }^{4}$, Gennaro Musi ${ }^{3}$, \\ Deliu-Victor Matei ${ }^{3}$, Ottavio De Cobelli ${ }^{3,5}$, Attila Tamas-Szora ${ }^{6}$, Cosmin Caraiani ${ }^{7}$, Andrei Lebovici ${ }^{8}$, \\ Flavia Epure ${ }^{9}$, Maria Bungardean $^{10}$, Radu-Tudor Coman ${ }^{11}$, Nicolae Crisan ${ }^{1,2}$ \\ ${ }^{1}$ Urology Department, Iuliu Hatieganu University of Medicine and Pharmacy, Cluj Napoca, Romania; ${ }^{2}$ Urology Department, Municipal Hospital, \\ Cluj Napoca, Romania; ${ }^{3}$ Department of Urology, IEO European Institute of Oncology, IRCCS, Milan, Italy; ${ }^{4}$ Medical Informatics and Biostatistics \\ Department, Iuliu Hatieganu University of Medicine and Pharmacy, Cluj Napoca, Romania; ${ }^{5}$ Department of Oncology and Hematology-Oncology, \\ Università degli Studi di Milano, Milan, Italy; ${ }^{6}$ Radiology Department, Municipal Hospital, Cluj Napoca, Romania; ${ }^{7}$ Medical Imaging Department, \\ Iuliu Hatieganu University of Medicine and Pharmacy, Cluj Napoca, Romania; ${ }^{8}$ Radiology Department, Iuliu Hatieganu University of Medicine \\ and Pharmacy, Cluj Napoca, Romania; ${ }^{9}$ Medical Imaging Department, Medisprof Cancer Center, Cluj Napoca, Romania; ${ }^{10}$ Pathology Department, \\ County Emergency Hospital, Cluj Napoca, Romania; ${ }^{11}$ Epidemiology Department, Iuliu Hatieganu University of Medicine and Pharmacy, Cluj \\ Napoca, Romania \\ Contributions: (I) Conception and design: E Cata, I Andras, M Ferro, N Crisan; (II) Administrative support: I Andras, D Leucuta, N Crisan; (III) \\ Provision of study materials or patients: E Cata, I Andras, A Tamas-Szora, C Caraiani, A Lebovici, F Epure, M Bungardean, RT Coman; (IV) \\ Collection and assembly of data: E Cata, I Andras, P Kadula, D Leucuta; (V) Data analysis and interpretation: E Cata, I Andras, M Ferro, P Kadula, \\ D Leucuta, G Musi, O De Cobelli, N Crisan; (VI) Manuscript writing: All authors; (VII) Final approval of manuscript: All authors. \\ \#These authors contributed equally to this work. \\ Correspondence to: Iulia Andras, MD, PhD. Urology Department, Iuliu Hatieganu University of Medicine and Pharmacy, 8 V Babes, 400012, Cluj- \\ Napoca, Romania. Email: dr.iuliaandras@gmail.com.
}

Background: Multiparametric magnetic resonance imaging (mpMRI) and targeted biopsy have become an integral part of the diagnosis of prostate cancer (PCa), as recommended by the European Association of Urology Guidelines. The aim of the current study was to evaluate the performance of MRI and MRItransrectal ultrasound (TRUS) fusion prostate biopsy as first biopsy setting in a tertiary center.

Methods: A cohort of 300 patients was included in the current analysis. All patients presented with clinical or biochemical suspicion of PCa and harbored at least one suspect lesion on mpMRI. MRI-TRUS fusion prostate biopsy, followed by 12 core systematic prostate biopsy were performed by the same operator using a rigid registration system.

Results: The mean age of the patients was 64 years (IQR: 58-68.5 years) and the mean PSA was $6.35 \mathrm{ng} / \mathrm{mL}$ (IQR: 4.84-9.46 ng/mL). Overall cancer and csPCa diagnosis rates were 47\% and 40.66\%. Overall $\mathrm{PCa} /$ csPCa detection rates were $20.4 \% / 11.1 \%, 52 \% / 45 \%$ and $68.5 \% / 66.7 \%$ for PI-RADS lesions 3,4 and 5 $(\mathrm{P}<0.001 / \mathrm{P}<0.0001)$. Larger lesion diameter and lesion volume were associated with $\mathrm{PCa}$ diagnosis $(\mathrm{P}=0.006$ and $\mathrm{P}=0.001$, respectively). MRI-TRUS fusion biopsy missed PCa diagnosis in 37 cases (of whom $48.6 \%$ ISUP 1) in comparison with 9 patients missed by systematic biopsy (of whom $11.1 \%$ ISUP 1). In terms of csPCa, systematic biopsy missed $77.7 \%$ of the tumors located in the anterior and transitional areas. The rate of csPCa was highest when targeted biopsy was associated with systematic biopsy: $86.52 \%$ vs. $68.79 \%$ for targeted biopsy $v$ s. $80.14 \%$ for systematic biopsy, $\mathrm{P}=0.0004$. In $60.6 \%$ of cases, systematic biopsy was positive for PCa at the same site as the targeted lesion. Of these patients, eight harbored csPCa and were diagnosed exclusively on systematic biopsy.

Conclusions: MRI-TRUS fusion prostate biopsy improves the diagnosis of csPCa. The main advantage of an MRI-guided approach is the diagnosis of anterior and transitional area tumors. The best results in terms of csPCa diagnosis are obtained by the combination of MRI-TRUS fusion with systematic biopsy. The 
systematic biopsy performed during MRI-targeted biopsy could have an important role in overcoming errors of MRI-TRUS fusion systems.

Keywords: Multiparametric magnetic resonance imaging (mpMRI); MRI-TRUS fusion prostate biopsy; prostate cancer diagnosis; systematic biopsy

Submitted Jun 15, 2020. Accepted for publication Sep 18, 2020.

doi: $10.21037 / \mathrm{tau}-20-1001$

View this article at: http://dx.doi.org/10.21037/tau-20-1001

\section{Introduction}

Prostate cancer $(\mathrm{PCa})$ is predicted to be the most frequent malignancy diagnosed in men in 2020 (1). In current practice, major changes occurred after the European Association of Urology recommendation to assess all men with suspicion for PCa by multiparametric magnetic resonance imaging (mpMRI) (2). A step forward was the possibility to perform targeted biopsy of the suspect lesions identified by mpMRI, in association with the gold standard 12-core systematic prostate biopsy (2).

The major advantage of mpMRI and targeted biopsy is improving the detection of clinically significant $\mathrm{PCa}$ (csPCa) and decreasing the diagnosis rate of insignificant $\mathrm{PCa}(3,4)$. Several prospective trials demonstrated that prostate biopsy could be avoided by mpMRI utilization in $27 \%$ to $49 \%$ cases $(3,5)$. Moreover, MRI-ultrasound (US) fusion biopsy significantly outperforms systematic biopsy in terms of cancer detection rate (CDR): $38 \%$ vs. $26 \%(\mathrm{P}=0.005)$ (4).

Despite all these, using mpMRI and targeted biopsy for PCa diagnosis is highly debatable especially in developing and emerging countries. Firstly, there is a high difference in access to proper imaging investigation, due to a lower number of MRI units available in Central and Eastern Europe compared to Western Europe (6). This limitation is even more acute when considering the unbalanced distribution of imaging resources between the public and private healthcare systems (7).

Secondly, there are high costs in the acquisition, maintenance and utilization of the technology necessary for mpMRI and MRI-targeted biopsy. MRI-US fusion and inbore targeted biopsy increase expenditure by 1.6 and 2.8 times, respectively, in comparison with systematic biopsy (8). Costs can be limited by the high negative predictive value of mpMRI, which could reduce the necessity to perform further tests (5) and by the higher diagnosis accuracy of targeted biopsy, which reduces further biopsies (9). By decreasing the overdiagnosis of low risk PCa, mpMRI and
MRI-guided biopsy limit the costs associated with further treatment and long term post-treatment complications care.

Thirdly, the quality of the imaging is essential for a proper radiologic interpretation (10). Prostate ImagingReporting and Data System guidelines (PI-RADS, last version v2.1) aimed to standardize the procedure among radiologists by specifying the minimal requirements for a good quality prostate mpMRI (11). Despite PI-RADS v2.1 guidance, mpMRI presents high sensitivity, but moderate specificity for csPCa (12). Currently, there is a challenge to provide the training and subspecialization in prostate mpMRI for sufficient number of radiologists in both developed and emerging countries health care systems (7).

The current study aims to evaluate the performance of mpMRI and MRI-US fusion targeted prostate biopsy in the real-life setting of a tertiary center in an emerging country. We present the current article in accordance with the START reporting checklist (available at http://dx.doi. org/10.21037/tau-20-1001) (13).

\section{Methods}

We analyzed our prospectively maintained database. Between October 2017 and February 2020, 400 patients underwent MRI-US fusion prostate biopsy by transrectal (TR) approach in our department. Of these, 300 patients had no history of prior biopsy and were included in the current analysis. The procedures were performed by two urologists. All patients had clinical (positive digital rectal examination) or biochemical suspicion of PCa (PSA $\geq 4 \mathrm{ng} / \mathrm{mL}$ ) and were assessed by mpMRI prior to the procedure. Antibiotic prophylaxis entailed quinolones starting two days before the biopsy. All procedures were performed with local anesthesia.

A number of 99 patients had performed mpMRI in our centre and 201 were referred to our service from other primary or secondary centers. In our centre, PI- 
RADS (v1-v2.1) Guidelines were used. MRI interpretation was provided by 3 radiologists with more than 5 years experience. Our protocol for mpMRI acquisition performed was previously detailed (14). The radiologists were aware of the clinical information of the patients. The minimal requirements for mpMRI performed in other centres were 1.5 $\mathrm{T}$ examination with $\mathrm{T} 2 \mathrm{WI}$, diffusion and contrastenhanced images and the radiologic description of suspected lesion (number of lesions, location, site, dimensions). PIRADS score was reported only for some of these patients $(\mathrm{n}=109)$. The remaining 92 patients harbored lesions considered suspicious by the radiologist (corresponding to a Likert score $\geq 3$ ), without receiving a PI-RADS score. Patients with suspicion for PCa but negative MRI were not included in the current analysis.

One to four biopsy cores were obtained first from each lesion by MRI-TRUS fusion biopsy, with a median number of 3 targeted cores/patient (IQR: 3-4). Additionally, a 12core systematic biopsy was performed. Both, targeted and systematic biopsies, were carried out by the same operator which was not blinded to the mpMRI result. All biopsies were performed using the Arietta 70a system (Hitachi, Japan) with endfire endorectal probe C41V1 2-10 mHz, RVS software and rigid registration, using sagittal and axial T2WI mpMRI sequences. The urologists delineated the suspect lesion based on radiologist description and drawing, when available.

The pathology evaluation was performed by 1 senior doctor (more than 20 years experience) and 2 young pathologists (3-5 years experience) under the supervision of the senior. Clinically significant PCa was defined as follows: (I) Gleason score of 7 or greater/ ISUP (International Society of Urological Pathology) grade 2 or higher, (II) tumor volume of $0.5 \mathrm{~cm}^{3}$ or greater, or a (III) positive extracapsular extension or seminal vesicle invasion. This definition of csPCa was employed taking into consideration that apart from ISUP grading, tumor volume correlates with the oncologic outcomes (2).

The study was conducted in accordance with the Declaration of Helsinki (as revised in 2013) and was approved by the local Hospital Ethical Committee (approval no. 8/20.02.2017). All patients enrolled completed and signed the informed consent form.

\section{Statistical analysis}

The statistical analysis was performed using $\mathrm{R}$ programme v.3.6.2. The continuous variables were presented as median and interquartile range, and as frequencies and proportions for categorical variables. Chi-square test was used to compare differences between categorical variables. Analysis of variance of continuous variables was performed using Kruskal-Wallis test. $\mathrm{P}<0.05$ was considered statistically significant.

\section{Results}

\section{General characteristics and cancer detection rate}

The clinical and pre-operative characteristics of the 300 patients included in our analysis are summarized in Table 1. The median age of the patients was 64 years (IQR $58-68.5$ years) and the median PSA was $6.35 \mathrm{ng} / \mathrm{mL}$ (IQR 4.84-9.46 ng/mL). In 204 patients mpMRI identified one lesion, whereas in 96 patients there were two or more suspicious lesions. The PI-RADS score was available for 208 patients (Table 1). The most frequent localization of the target lesions was in the peripheral area $(64 \%)$, while the rest were located in the anterior $(3 \%)$ and transitional areas (28.66\%).

The overall CDR and csPCa diagnostic rate were $47 \%$ (141 patients) and 40.66\% (122 patients), respectively. Patients diagnosed with high-grade intraepithelial neoplasia or suspicious lesions but not adenocarcinoma (atypical small acinar proliferation of prostate) according to the contemporary diagnostic criteria were not considered positive for the outcome of interest (PCa). Of the patients confirmed with PCa, $76.5 \%$ harbored disease with ISUP grading $\geq 2$, as follows: ISUP grade 2: 77 patients (54.6\%), ISUP grade $3: 23$ patients (16.3\%), ISUP grade $4: 4$ patients $(2.8 \%)$ and ISUP grade $5: 4$ patients $(2.8 \%)$.

\section{Added value of MRI-TRUS fusion and systematic biopsy}

MRI-TRUS fusion targeted biopsy missed $37 \mathrm{PCa}$ patients $(26.2 \%$, of whom 25 were csPCa), as compared to systematic biopsy-9 cases $(6.4 \%$, all were csPCa). The maximum diameter of the lesion, lesion volume and the site within the prostate did not influence the diagnostic ability of MRI-US fusion biopsy (Table 2). Still, we observed that systematic biopsy lost lesions with higher diameter and higher volume.

The rate of clinically significant disease diagnosis was $80.14 \%$ for the patients confirmed with PCa by systematic biopsy, $68.79 \%$ for targeted biopsy and $86.52 \%$ for their combination, $\mathrm{P}=0.0004$. We analyzed the differences in 
Table 1 General characteristics of the patients

\begin{tabular}{|c|c|}
\hline Characteristic & Outcome \\
\hline Age (years), median (IQR) & $64(58-68.5)$ \\
\hline PSA (ng/mL), median (IQR) & $6.35(4.84-9.46)$ \\
\hline Prostate volume (g), median (IQR) & $47(37.44-67.3)$ \\
\hline Digital rectal examination, positive & 100 patients $(33.3 \%)$ \\
\hline \multirow[t]{2}{*}{ Single/multiple lesions } & $\begin{array}{l}\text { Single lesion: } 204 \text { patients } \\
(68 \%)\end{array}$ \\
\hline & $\begin{array}{l}\text { Multiple lesions: } 96 \\
\text { patients (32\%) }\end{array}$ \\
\hline \multirow[t]{4}{*}{ Location of suspicious lesions } & Anterior: 9 patients $(3 \%)$ \\
\hline & $\begin{array}{l}\text { Peripheral: } 192 \text { patients } \\
(64 \%)\end{array}$ \\
\hline & $\begin{array}{l}\text { Transitional: } 86 \text { patients } \\
(28.66 \%)\end{array}$ \\
\hline & $\begin{array}{l}\text { Diffuse lesions: } 13 \text { patients } \\
(4.33 \%)\end{array}$ \\
\hline \multirow[t]{4}{*}{ Site of suspicious lesions } & Apex: 57 patients (23.33\%) \\
\hline & $\begin{array}{l}\text { Mid-gland: } 109 \text { patients } \\
(36.33 \%)\end{array}$ \\
\hline & Base: 114 patients (38\%) \\
\hline & $\begin{array}{l}\text { Diffuse lesions: } 20 \text { patients } \\
(6.66 \%)\end{array}$ \\
\hline $\begin{array}{l}\text { Maximum diameter of the suspicious } \\
\text { lesion }(\mathrm{mm}) \text {, median }(\mathrm{IQR})\end{array}$ & $13(10-17)$ \\
\hline $\begin{array}{l}\text { Volume of the lesion }\left(\mathrm{cm}^{3}\right) \text {, median } \\
\text { (IQR) }\end{array}$ & $0.64(0.31-1.32)$ \\
\hline \multirow[t]{3}{*}{ PI-RADS score } & 3: 54 patients $(26 \%)$ \\
\hline & 4: 100 patients $(48.1 \%)$ \\
\hline & 5: 54 patients $(26 \%)$ \\
\hline
\end{tabular}

$I Q R$, interquartile range; PI-RADS, prostate imaging and reporting data system; PSA, prostate specific antigen.

ISUP grading between MRI-TRUS fusion biopsy and systematic biopsy cores. The rate of upgrading to a higher ISUP grade disease was $22.1 \%$ (21 patients) for systematic biopsy and $18.9 \%$ (18 patients) for targeted biopsy. In 59\% of the patients there was no upgrading to a more aggressive disease by either type of biopsy. We did not identify any significant predictors for the occurrence of upgrading (PSA $\mathrm{P}=0.499$, site of lesion $\mathrm{P}=0.963$, lesion dimension $\mathrm{P}=0.66$, lesion volume $\mathrm{P}=0.903$, $\mathrm{PI}-\mathrm{RADS}$ score $\mathrm{P}=0.268$ ), except the location of the lesion $(\mathrm{P}=0.01)$. For lesions located in the peripheral area, the rate of upgrading was $18.1 \%$ and $16.7 \%$ by systematic and targeted biopsy, respectively. For lesions located in the transitional area, the rate of upgrading was $16.7 \%$ and $41.7 \%$ and for lesions located in the anterior area $75 \%$ and $0 \%$ by systematic and targeted biopsy, respectively.

\section{Factors associated with PCa diagnosis}

The overall and clinically significant CDR according to the pre-operative PI-RADS score was as follows: PI-RADS $3-20.4 \%$ overall and $11.1 \%$ csPCa, PI-RADS 4-52\% overall and $45 \%$ csPCa, PI-RADS $5-68.5 \%$ overall and $66.7 \%$ csPCa $(\mathrm{P}<0.001$ and $\mathrm{P}<0.0001$, respectively). Higher overall rates of $\mathrm{PCa}$ were identified in anterior (77.8\%) lesions as compared to those located in the peripheral (53.6\%) and transitional areas (27.9\%), $\mathrm{P}=0.0006$. Similarly, higher rates of csPCa were observed in anterior $(66.7 \%)$ vs. peripheral (45.8\%) and transitional $(24.4 \%)$ lesions, $\mathrm{P}=0.0009$.

The site of the lesion within the prostate (apex/midgland/base) was not associated with $\mathrm{PCa}$ diagnosis $(\mathrm{P}=0.16$ for $\mathrm{PCa}$ and $\mathrm{P}=0.63$ for csPCa). Overall, $\mathrm{PCa}$ was more frequently diagnosed in patients with larger lesions: median diameter of lesion was $14 \mathrm{~mm}$ (IQR: 10.5-18) in PCa patients vs. $11.5 \mathrm{~mm}$ (IQR: 9-15) in $\mathrm{BPH}$ cases, $\mathrm{P}=0.002$. The same was true for aggressive PCa (ISUP grade $\geq 2$ ): median diameter of lesion $14 \mathrm{~mm}$ (IQR: 11-18) vs. $11 \mathrm{~mm}$ (IQR: 9-15) for ISUP 1 disease or $\mathrm{BPH}, \mathrm{P}=0.001$. Similarly, lesion volume was correlated with overall $\mathrm{PCa}$ diagnosis (median volume of $0.85 \mathrm{~cm}^{3}$ for PCa vs. $0.49 \mathrm{~cm}^{3}$ for $\mathrm{BPH}$, $\mathrm{P}=0.001)$ and with the presence of a disease with ISUP grade $\geq 2$ (median volume of 0.85 vs. $0.5 \mathrm{~cm}^{3}$ for ISUP 1 , $\mathrm{P}=0.009)$.

\section{Impact of lesion location and errors of targeting}

Of the patients with PCa missed by targeted biopsy, 78.4\% harbored peripheral lesions. On the other hand, of the patients missed by systematic biopsy $77 \%$ had anterior and transitional lesions (Table 2).

When analyzing only the patients with clinically significant $\mathrm{PCa}$ who were lost for diagnosis, we observed that $77.7 \%$ of the patients missed by systematic biopsy harbored tumors located in the anterior and transitional areas (Table 3). On the contrary, $67.85 \%$ of the patients with csPCa lost by targeted biopsy had lesions located in the peripheral area. None of the patients lost by MRI-TRUS 
Table 2 Characteristics of the patients lost for diagnosis by both biopsy methods

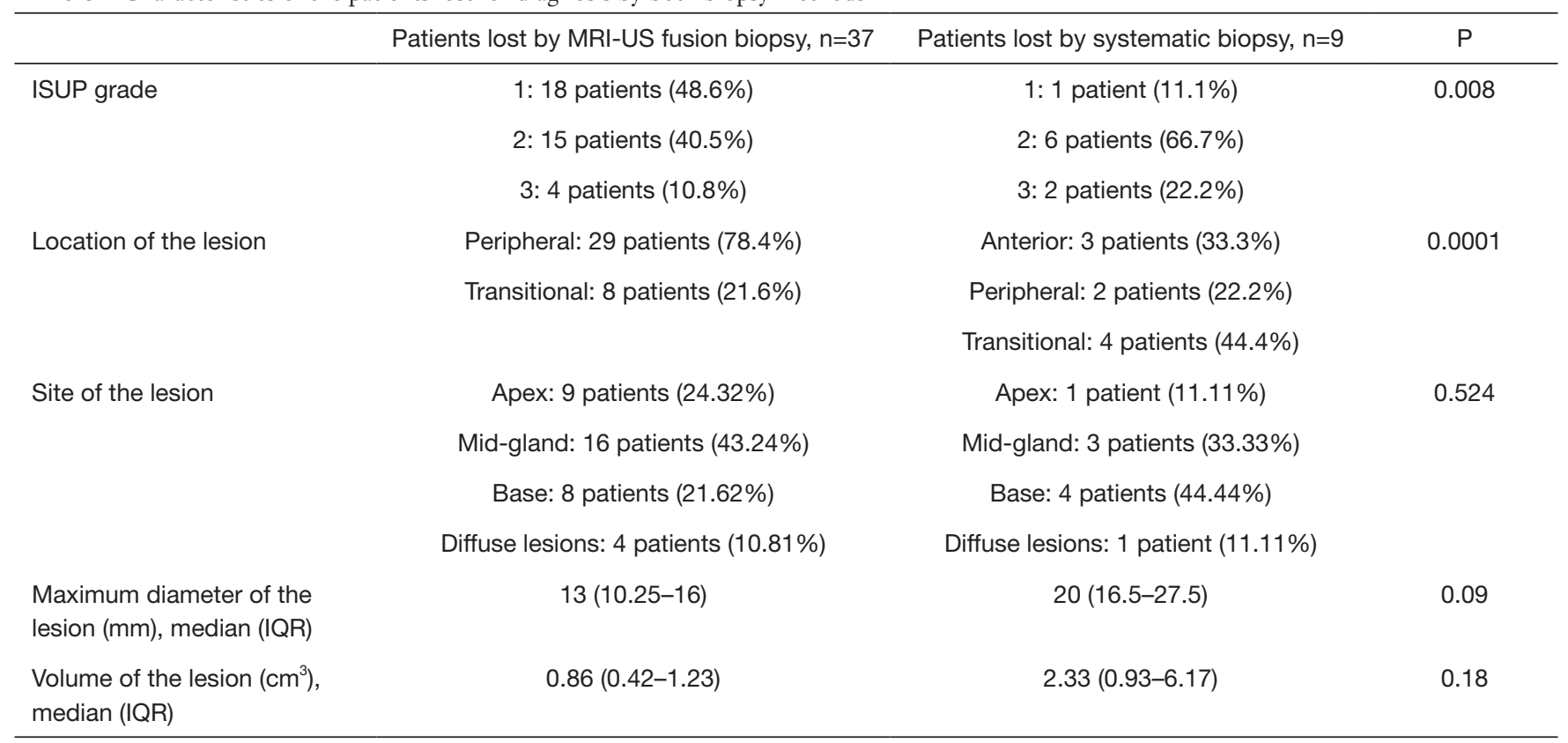

IQR, interquartile range; ISUP, International Society of Urological Pathology; MRI, magnetic resonance imaging; US, ultrasound.

Table 3 Characteristics of the patients with clinically significant PCa lost for diagnosis

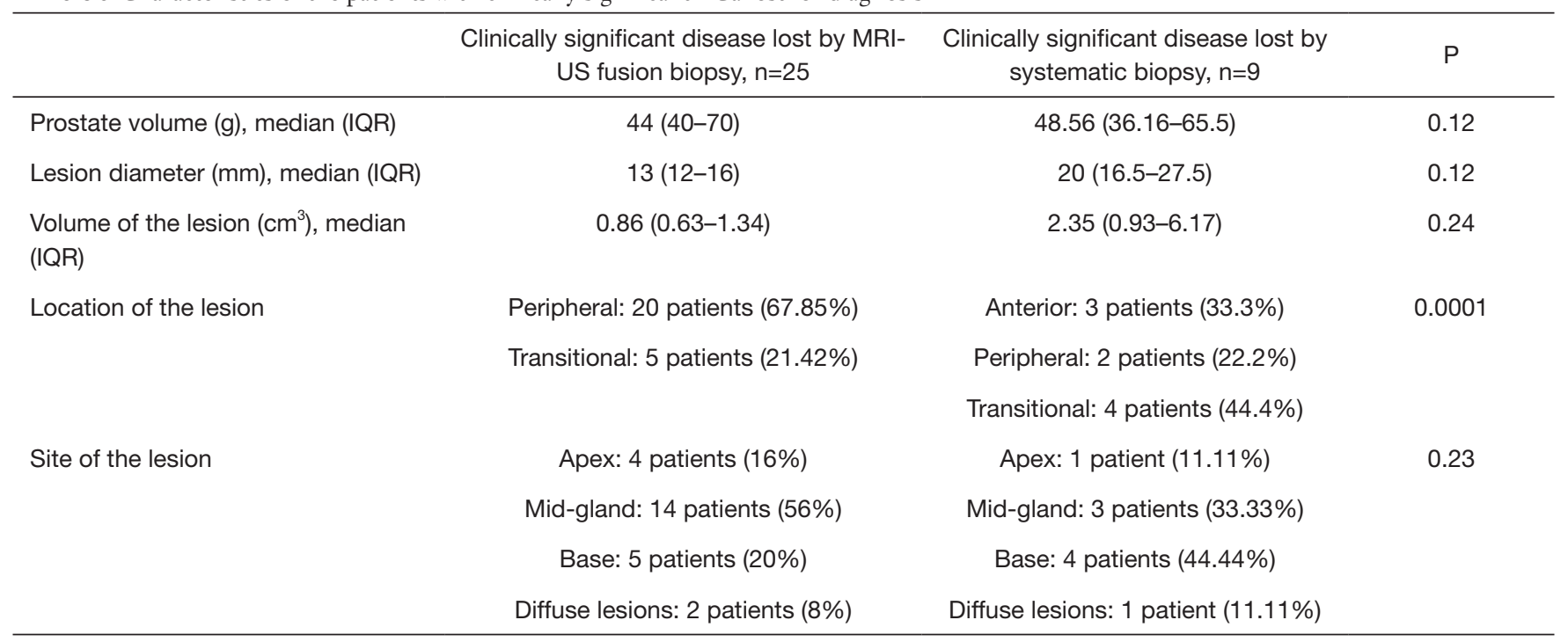

IQR, interquartile range; MRI, magnetic resonance imaging; US, ultrasound.

fusion biopsy harbored anterior lesions (Table 3).

In $60.6 \%$ of cases (80 patients), systematic biopsy was positive for $\mathrm{PCa}$ at the same site of the prostate (base/midgland/apex) as the targeted lesion. Of these patients, $17.5 \%$ (14 patients) were diagnosed exclusively on systematic biopsy. These cases represent the true targeting error.
The median volume of the lesion for these patients was $0.97 \mathrm{~cm}^{3}$ (IQR 0.59-2.31) and all lesions were located in the peripheral area. Eight patients (2.6\% of the whole study group) diagnosed with PCa only by systematic biopsy at the same site where the MRI-TRUS fusion biopsy was negative harbored csPCa. 


\section{Complications}

The readmission rate for post-biopsy sepsis in our cohort was $2 \%$. No readmissions were encountered for other complications.

\section{Discussion}

The main objective of our study was to assess the performance of MRI-US fusion prostate biopsy for the diagnosis of PCa in a tertiary hospital in an emerging economy country. Our initial findings regarding overall PCa diagnosis (47\%) and csPCa (40.66\%) are comparable to the data coming from reference centers $(5,15,16)$. The advantage of an MRI-TRUS fusion approach for prostate biopsy resides mostly in the diagnosis of anterior and transitional PCa. As seen in our series, $77.7 \%$ of the $\mathrm{PCa}$ patients missed by systematic biopsy harbored anterior and transitional lesions, which were diagnosed using a targeted approach. On the other hand, $78.4 \%$ of the patients missed by MRI-TRUS fusion biopsy harbored peripheral lesions, which can easily be diagnosed within a systematic biopsy. Moreover, we identified 14 patients diagnosed with $\mathrm{PCa}$ exclusively by systematic biopsy at the same site as the lesion, which we considered as true targeting error. Thus, MRI-targeted cores improve the diagnosis rate when added to systematic biopsy, while the systematic cores performed during an MRI-TRUS fusion biopsy can overcome errors of registration and targeting. As such, we consider that at least for the current moment, the combination of MRItargeted and systematic biopsy should be the recommended approach.

We observed a statistically significant correlation between the PI-RADS score and the detection rate of PCa. The rate of overall and csPCa diagnosis increased with higher PI-RADS score. Thus, the PI-RADS score has become an important adjunct in the estimation of PCa risk before biopsy. This information from mpMRI can assist patient counselling prior to an invasive procedure, such as prostate biopsy, contributing to an informed decision making. The accuracy of mpMRI interpretation and PI-RADS score grading are linked to the radiologist's experience, but progress has been made after the implementation of PI-RADS v2.1 with study showing improved interreader agreement (17).

The main advantage of an MRI-US fusion approach is the sampling of anterior and transitional tumors. In our study, approximately $77.7 \%$ of overall and csPCa lost by systematic biopsy were located in these areas. Systematic prostate biopsy samples the posterior area of the prostate, whereas tumors located in the transitional or anterior areas are usually missed. Several studies showed that in patients with multiple negative biopsies there is an increased risk (up to $70 \%$ ) to harbor an anterior csPCa $(18,19)$. As such, these patients are exposed to a greater complications risk and there is a chance to miss the therapeutic window if they are not compliant to repeat this procedure. An MRIguided approach from the start could reduce the number of unnecessary invasive procedures. Furthermore, losing peripheral lesions by MRI-US fusion biopsy can safely be overcome by adding systematic biopsy.

Considering the high accuracy of mpMRI and MRItargeted biopsy, it has been advocated that systematic biopsy will become obsolete. Yet, multiple studies have shown the added value of each in terms of diagnosis of csPCa and Gleason score accuracy $(20,21)$. Indeed, our series confirm that the association of MRI-targeted to systematic biopsy leads to a more accurate diagnosis of csPCa: $80.14 \%$ for systematic biopsy vs. $68.79 \%$ for targeted biopsy $v$ s. $86.52 \%$ for the combination. Several authors emphasized the absolute necessity of systematic biopsy, providing data showing that the targeted biopsy can miss up to $20 \%$ of csPCa (22-24). Also, Dell'Oglio et al. (25) evaluated the possibility to avoid systematic biopsy when performing an MRI-targeted approach. Using a risk prediction model for the presence of csPCa outside the index lesion, the authors did not identify a subpopulation in whom systematic biopsy could safely be avoided. On the other hand, in a multicenter analysis of 640 patients—of whom 357 underwent targeted and systematic biopsy, Miah et al. (26) showed that the added value of systematic biopsy for the detection of csPCa was minimal $(0.8 \%)$. These differences could be explained by several details: firstly, the authors report taking twice as many cores/target as compared to our study (4-6 cores/ target $v s .3$ cores/target). Furthermore, no details are provided by the authors regarding the number of cores corresponding to the systematic biopsy. The biopsies were performed by a transperineal approach as compared to transrectal approach in our study and included also patients in second biopsy setting, which may account for a better detection rate of the transperineal approach. Last, but not least, different definitions of csPCa are another bias when aiming to compare different series. On the other hand, the saturation biopsy of MRI-targeted lesion was analyzed as a potential alternative to concurrent systematic biopsy. Hansen et al. (27) assessed the number of optimal cores associated to mpMRI targeted biopsy in order to have 
an accurate PCa diagnosis. The authors observed that a number of 2 supplementary cores per each sector adjacent to the suspected lesion leads to the diagnosis of more than $90 \%$ of csPCa cases identified by the combination of targeted and systematic biopsy.

Taking into consideration opposing evidence, the decision to perform systematic biopsy in addition to MRItargeted is still debatable, depending on multiple factors such as the centre, quality of MRI or number of targeted cores. As such, most probably systematic and targeted biopsies will still be used as complementary procedures.

In general practice, MRI-targeted and systematic biopsy are performed by the same operator, not blinded to the mpMRI result. Thus, there is the possibility to use the mpMRI information not only for the targeted cores, but also to guide the systematic biopsy. As a result, the systematic biopsy becomes cognitive-guided. The purpose of this approach is to improve the cancer diagnosis rate by overcoming errors of registration and targeting. This can be particularly important for rigid registration systems, as they do not account for prostate deformation during the procedure. We consider that the good performance of systematic biopsy in the current study is the result of the fact that the operator was aware of the localisation of the suspect lesion and performed cognitive guided systematic biopsy. We observed that in $60.6 \%$ of cases systematic biopsy was positive at the same site as the targeted lesion. Furthermore, we observed that in 8 patients cognitiveguided systematic biopsy overcame the error of MRITRUS fusion registration and diagnosed csPCa. Other authors confirm our experience, as Miah et al. (28) showed that in $77.77 \%$ of patients in whom the diagnosis of csPCa was missed by MRI-targeted biopsy, systematic biopsy cores confirmed the disease in the same sector as the target lesion.

The strength of our study is represented by the illustration of real-life setting, with mpMRI performed in centers with various experience. We also consider that a reduced number of physicians (two) to perform MRITRUS fusion biopsy leads to a low heterogeneity in the results caused by different learning curves, as observed also in other centers $(29,30)$.

The limitations of our study consist in the fact that targeted and systematic biopsy were performed by the same operator, not blinded to the mpMRI result. This precluded an objective comparison between the two biopsy methods. Also, the fact that one third of the mpMRI was not evaluated according to the PI-RADS criteria and data regarding radiologists experience lacked, might have affected the performance of mpMRI and subsequently of targeted biopsy. Another potential limitation of our study is the transrectal route for performing the biopsy, in the context of the recent movement towards transperineal access route, with regards to decreased complications rate. Nonetheless, none of the PCa cases missed by MRI-TRUS fusion biopsy in our cohort had lesions located in the anterior area.

\section{Conclusions}

In real-life setting, MRI-TRUS fusion prostate biopsy improves the diagnosis of csPCa irrespective of the limitations of an emerging economy healthcare system. The main advantage of an MRI-guided approach is the diagnosis of anterior and transitional area tumors. The best results in terms of csPCa diagnosis and Gleason score are obtained by the combination of MRI-TRUS fusion with systematic biopsy. The systematic biopsy performed during MRItargeted biopsy is cognitive-guided, thus it could have an important role in overcoming errors of MRI-TRUS fusion systems.

\section{Acknowledgments}

Funding: The study was supported by a grant from the Romanian Ministry of Education and Research, CNCS UEFISCDI, project no. PN-III-P1-1.1-PD-2019-1237, within PNCDI III.

\section{Footnote}

Reporting Checklist: The authors have completed the START reporting checklist. Available at http://dx.doi.org/10.21037/ tau-20-1001

Data Sharing Statement: Available at http://dx.doi. org/10.21037/tau-20-1001

Peer Review File: Available at http://dx.doi.org/10.21037/ tau-20-1001

Conflicts of Interest: All authors have completed the ICMJE uniform disclosure form (available at http://dx.doi. org/10.21037/tau-20-1001). The authors have no conflicts of interest to declare

Ethical Statement: The authors are accountable for all 
aspects of the work in ensuring that questions related to the accuracy or integrity of any part of the work are appropriately investigated and resolved. The study was conducted in accordance with the Declaration of Helsinki (as revised in 2013) and was approved by the local Hospital Ethical Committee (approval no. 8/20.02.2017). All patients enrolled completed and signed the informed consent form.

Open Access Statement: This is an Open Access article distributed in accordance with the Creative Commons Attribution-NonCommercial-NoDerivs 4.0 International License (CC BY-NC-ND 4.0), which permits the noncommercial replication and distribution of the article with the strict proviso that no changes or edits are made and the original work is properly cited (including links to both the formal publication through the relevant DOI and the license). See: https://creativecommons.org/licenses/by-nc-nd/4.0/.

\section{References}

1. American Cancer Society. Cancer Facts \& Figures 2020.

2. Mottet N, van den Bergh RCN, Briers E, et al. EAUEANM-ESTRO-ESUR-SIOG Guidelines on Prostate Cancer. Available online: https://uroweb.org/wp-content/ uploads/EAU-EANM-ESUR-ESTRO-SIOG-Guidelineson-Prostate-Cancer-2019.pdf

3. van der Leest $M$, Cornel E, Israël B, et al. Head-to-head comparison of transrectal ultrasound-guided prostate biopsy versus multiparametric prostate resonance imaging with subsequent magnetic resonance-guided biopsy in biopsy-naïve men with elevated prostate-specific antigen: a large prospective multicenter clinical study. Eur Urol 2019;75:570-8.

4. Kasivisvanathan V, Rannikko AS, Borghi M, et al. MRItargeted or standard biopsy for prostate-cancer diagnosis (Precision Study). N Engl J Med 2018;378:1767-77.

5. Ahmed HU, El-Shater Bosaily A, Brown LC, et al. Diagnostic accuracy of multi-parametric MRI and TRUS biopsy in prostate cancer (PROMIS): a paired validating confirmatory study. Lancet 2017;389:815-22.

6. Denjoy N. European Coordination Committee of the Radiological, Electromedical, and Healthcare IT Industry: Medical imaging equipment age, profile \& density. Available online: http://www.cocir.org/uploads/ media/16052_COC_AGE_PROFILE_web_01.pdf (2016).

7. Schlemmer HP, Bittencourt LK, D'Anastasi M, et al. Global challenges for cancer imaging. J Glob Oncol 2018;4:1-10.
8. Venderink W, Govers TM, De Rooij M, et al. Costeffectiveness comparison of imaging-guided prostate biopsy techniques: Systematic transrectal ultrasound, direct in-bore MRI, and image fusion. AJR Am J Roentgenol 2017;208:1058-63.

9. Drost FH, Osses DF, Nieboer D, et al. Prostate MRI, with or without MRI-targeted biopsy, and systematic biopsy for detecting prostate cancer. Cochrane Database Syst Rev 2019;4:CD012663.

10. Engels RRM, Israël B, Padhani AR, et al. Multiparametric magnetic resonance imaging for the detection of clinically significant prostate cancer: what urologists need to know. Part 1: acquisition. Eur Urol 2020;77:457-68.

11. Turkbey B, Rosenkrantz AB, Haider MA, et al. Prostate Imaging Reporting and Data System Version 2.1: 2019 Update of Prostate Imaging Reporting and Data System Version 2. Eur Urol 2019;76:340-51.

12. Zhen L, Liu X, Yegang C, et al. Accuracy of multiparametric magnetic resonance imaging for diagnosing prostate Cancer: A systematic review and metaanalysis. BMC Cancer 2019;19:1244.

13. Moore CM, Kasivisvanathan V, Eggener S, et al. Standards of reporting for MRI-targeted biopsy studies (START) of the prostate: Recommendations from an international working group. Eur Urol 2013;64:544-52.

14. Andras I, Crisan D, Cata E, et al. MRI-TRUS fusion guided prostate biopsy - Initial experience and assessment of the role of contralateral lobe systematic biopsy. Med Ultrason 2019;21:37-44.

15. Ahdoot M, Wilbur AR, Reese SE, et al. MRI-targeted, systematic, and combined biopsy for prostate cancer diagnosis. N Engl J Med 2020;382:917-28.

16. Rastinehad AR, Turkbey B, Salami SS, et al. Improving detection of clinically significant prostate cancer: Magnetic resonance imaging/transrectal ultrasound fusion guided prostate biopsy. J Urol 2014;191:1749-54.

17. Brembilla G, Dell'Oglio P, Stabile A, et al. Interreader variability in prostate MRI reporting using Prostate Imaging Reporting and Data System version 2.1. Eur Radiol 2020;30:3383-92.

18. Volkin D, Turkbey B, Hoang AN, et al. Multiparametric magnetic resonance imaging (MRI) and subsequent $\mathrm{MRI} /$ ultrasonography fusion-guided biopsy increase the detection of anteriorly located prostate cancers. BJU Int 2014;114:E43-9.

19. Schouten MG, Hoeks CMA, Bomers JGR, et al. Location of prostate cancers determined by multiparametric and MRI-guided biopsy in patients with elevated prostate- 
specific antigen level and at least one negative transrectal ultrasound-guided biopsy. AJR Am J Roentgenol 2015;205:57-63.

20. Rouvière $\mathrm{O}$, Puech $\mathrm{P}$, Renard-Penna $\mathrm{R}$, et al. Use of prostate systematic and targeted biopsy on the basis of multiparametric MRI in biopsy-naive patients (MRIFIRST): a prospective, multicentre, paired diagnostic study. Lancet Oncol 2019;20:100-9.

21. Siddiqui MM, Rais-Bahrami S, Turkbey B, et al. Comparison of MR/ultrasound fusion-guided biopsy with ultrasound-guided biopsy for the diagnosis of prostate cancer. JAMA 2015;313:390-7.

22. Mortezavi A, Märzendorfer O, Donati OF, et al. Diagnostic accuracy of multiparametric magnetic resonance imaging and fusion guided targeted biopsy evaluated by transperineal template saturation prostate biopsy for the detection and characterization of prostate cancer. J Urol 2018;200:309-18.

23. Maxeiner A, Kittner B, Blobel C, et al. Primary magnetic resonance imaging/ultrasonography fusion-guided biopsy of the prostate. BJU Int 2018;122:211-8.

24. Preisser F, Theissen L, Wenzel M, et al. Performance of combined magnetic resonance imaging/ultrasound fusionguided and systematic biopsy of the prostate in biopsynaïve patients and patients with prior biopsies. Eur Urol Focus 2019;49:2-9.

25. Dell'Oglio P, Stabile A, Soligo M, et al. There is no

Cite this article as: Cata E, Andras I, Ferro M, Kadula P, Leucuta D, Musi G, Matei DV, De Cobelli O, Tamas-Szora A, Caraiani C, Lebovici A, Epure F, Bungardean M, Coman RT, Crisan N. Systematic sampling during MRI-US fusion prostate biopsy can overcome errors of targeting-prospective single center experience after 300 cases in first biopsy setting. Transl Androl Urol 2020;9(6):2510-2518. doi: 10.21037/tau-20-1001 way to avoid systematic prostate biopsies in addition to multiparametric magnetic resonance imaging targeted biopsies. Eur Urol Oncol 2020;3:112-8.

26. Miah S, Hosking-Jervis F, Connor MJ, et al. A multicentre analysis of the detection of clinically significant prostate cancer following transperineal image-fusion targeted and nontargeted systematic prostate biopsy in men at risk. Eur Urol Oncol 2020;3:262-9.

27. Hansen NL, Barrett T, Lloyd T, et al. Optimising the number of cores for magnetic resonance imaging-guided targeted and systematic transperineal prostate biopsy. BJU Int 2020;125:260-9.

28. Miah S, Servian P, Patel A, et al. A prospective analysis of robotic targeted MRI-US fusion prostate biopsy using the centroid targeting approach. J Robot Surg 2020;14:69-74.

29. Stabile A, Dell'Oglio P, Gandaglia G, et al. Not All Multiparametric Magnetic Resonance Imagingtargeted Biopsies Are Equal: The Impact of the Type of Approach and Operator Expertise on the Detection of Clinically Significant Prostate Cancer. Eur Urol Oncol 2018;1:120-8.

30. Meng X, Rosenkrantz AB, Huang R, et al. The Institutional Learning Curve of Magnetic Resonance Imaging-Ultrasound Fusion Targeted Prostate Biopsy: Temporal Improvements in Cancer Detection in 4 Years. J Urol 2018;200:1022-9. 